\title{
LECTURE ALCHIMIQUe D'UN POEME D'ANNE hEBERT
}

\author{
Zília Mara Scarpari Schmidt \\ Universidade Federal do Paraná
}

\section{RESUM E}

A partir de l'analyse sémantique du poème Alchimio du Jour, d' Anne Hébert, on aboutit à une lecture symbolique où l'on reconnait toutes les étapes d'un véritable processus alchimique. Outre la lecture référentielle de la naissance du jour, trois autres isotopies enchevêtrées peuvent être perçues au cours de l'analyse: l'alchimie physique, l'alchimie mystique et l'alchimie pótique.

\section{ALCHIMIE DU JOUR, Anne Hébert.}

1 - Qu'aucune servante ne te serve en cej jour où tu lias ta peine sauvage, bête de sang aux branches basses du noir sapin,

2 - Ne le dites pas aux filles de feux roux, ne prévenez pas les filles aux cœurs violets;

3 - Elles paraitraient toutes les sept en ta chambre portant les pitiés bleues en des amphores traquilles hissées sur leurs cheveux,

4 - Elles glisseraient la longue file de leurs ombres mauves pareilles à l'envers des flammes marines en une calme frise-processionnelle aux quatre vents de tes murs.

5 - Ne prévenez pas les filles aux pieds de feutre vert decoupés-a même d'antiques tapis réservés au déroulement lent des douleur sacrées, pré doux au soleil tondu, aux herbes silencieuses et drues sans l'espace vif du cri,

6 - Ni l'obscure ef forte vibration de l'amour souterrain semblable à la passion excessive de la mer en l'origine de son chant appareillant.

7 - La première fille alertée joindrait ses sœurs, une à une, ef leur parlerait bas de l'amour blessé amarré aux feuillages de tes veines ouvertes,

8 - La plus sombre des soeurs désignées te porterait des baumes nouvellement fleuris sur des cœurs amers, très vieux celliers désaffectés, plate-bande des remèdes et des conseils nocturnes. 
9 - Tandis que la plus lente d'entre elles referait son visage de larmes brûlées comme une belle pierre mise à jour sous des fouilles patientes et pures,

10 - La voici que délègue vers toi une fille de sel portant des paniers fins pour ses moissons claires. Elle soupèse en chemin le poids de tes pleurs cueillis à la pointe de l'ongle comme la rosée sur le jardin qui s'affale,

11 - Vois, celle qui a nom Véronique plie de grandes toiles pures et rêve d'un visage à saisir en sa grimace à même des voiles déroulées comme de clairs miroirs d'eau,

12 - Se hâte la fille-fièvre parée d'épines cuivrées, maintenant que la nuit, en sa haute taille levée, bouge ses paumes mûres comme de noirs tournesols,

13 - Sur tes paupières bientôt elle posera ses mains étroitement comme des huitres vives où la mort médite, des siècles de songe sans faille, la blanche floraison d'une perle dure

14 - O toi qui trembles dans le vent, ayant hissé la beauté de ton visage au mât des quatre saisons,

15 - Toi qui grinces de sable, ointe par des huiles pures, nue, en des miracles certains de couleur agile ef d'eau puissante,

16 - Redoute l'avènement silencieux des compassions crayeuses aux faces d'argiles brouillés;

17 - Pose le vert contre le bleu, usant d'un vif pouvoir, ne crains pas l'ocre sur le pourpre, laisse débonder le verbe se liant au monde telle la flèche à son arc,

18 - Laisse le don alerté mûrir son étrange alchimie en des équipages fougueux,

19 - Profère des choses sauvages dans le soleil, nomme toute chose face au tumulte des grands morts friables et irrités.

20 - Les murs aux tessons bleus crèvent comme des cercles d'eau sur la mer,

21 - Et le point du cœur dessine sa propre souple ceinture,

22 - Le jour, pour la seconde fois convoqué, monte en parole comme un large pavot éclatant sur sa tige. 


\section{1 - INTRODUCTION}

Anne Hébert, poète canadien, nous offre dans Alchmie du Jour, le produit final du Grand Magistère poétique. En fait, la fonction déictique et métalinguistique du titre du poème nous annonce une lecture où l'on pourra suivre les pas d'un véritable processus alchimique.

L'ancienne Alchimie était l'art de la transmutation des métaux devant aboutir à la découverte de la Pierre philosophale, la quintessence des choses. Celle-ci permettait la projection, c'est-à-dire, l'opération consistant à transformer un métal vil en or; si elle était capable de "régénerer les métaux lépreux" en or, c'est qu'elle avait des propriétés médicales qui pouvaient rendre da santé aux malades, éliminer toutes les impuretés de la matière, bref régénérer tous les êtres imparfaits. C'est ainsi que l'alchimie pratique devient une mystique: toouver la Pierre philosophale, c'est trouver la panacée pour tous les maux, découvrir le véritable bonheur, posséder la Connaissance parfaite, accéder à l'Absolu. L'homme devient la ma. tière même du Grand Oeuvre, défini alors comme l'union en Dieu far l'extase. II faut bien insister, cependant, que l'adepte pratique simultanément l'OEuvre mystique et l'OEuvre physique, qui sont analogues et parallèles. La description de l'OEuvre physique s'adapte :Irictement aux opérations de l'OEuvre spirituel ef vice-versa.

Le travail de l'alchimiste pouvait être réduit à quatre phoses principales: la purification du sujet, la préparation de la matière de la Pierre, la cuisson dans l'Oeuf philosophique ef la préparation de la Pierre philosophale.

La matière de la Pierre naissait du "mariage philosophique" du soufre, principe masculin, symbole de l'âme, et du mercure, principe féminin, symbole du corps. Le sel, comparé à l'esprit vital qui unit l'âme au corps, était le moyen d'union entre les deux éléments.

Aussitôt le feu allumé dans l'Athanor, le Grand OEuvre proprement dit commençait. A l'intérieur de l'oeuf philosophique, la matière se transformait progressivement, prenant diverses colora. tions. Après la phase designée sous le nom de putréfaction, la pierre devenait progressivement blanche: c'était la résurreetion. Si on arrê. lait l'OEuvre à ce point, on obtenait la Pierre blanche, capable de changer les métaux en argent. Enfin, après avoir passé par toutes les couleurs de l'arc-en-ciel, la Pierre devenait d'un rouge éclałant: cétait la rubification ou sublimation. On brisait l'oeuf philosophique, or possédait maintenant la Pierre philosophale, rouge et parfaite. 
Mais, avant d'être utilisable, elle devait subir encore une préparation, designée sous le nom de fermentation: la Pierre, masse friable rouge, était mêlée à de l'or fondu et augmentaif ainsi indéfiniment en qualité et en quantité.

Bref, enfermé dans l'Oeuf, la matière meurt, pourrit, puis renait. D'ou l'association de la Pierre au Christ:

Ce monarque, libéré du tombeau (...). lui qui ressuscite, Phoenix, pourrit, Grain, nourrit, Pélican, de son sang ses fils spirituels, nait, Salamandre, du feu de l'Esprit, ef le reçoit, et s'en restaure, ce monarque n'est-il pas JésusChrist? ${ }^{1}$

(Philalethe)

Nous avons cru nécessaire cette explication préalable pour mieux saisir le texte d'Anne Hébert. En fait, Alchimie du jour est en principe une suite d'opérations alchimiques où les transmutations mystiques se superposent aux transformations physiques. Dans ces processus le mythe du Christ se réécrit.

"L'Alchimie est envisagée comme une technique du salut, qui vise à délivrer l'etincelle de la lumière éternelle tombée dans les ténèbres de la matière." " Voilà comment s'établit dans Alchimie du Jour l'isomorphisme entre atchimie, jour et lumière. Si jour connote tout naturrellement avec lumière, celle-ci, en tant que symbole fon. damental du monde manifesté, principe même de la vie et expresson visible du Verbe divin, se relie à la parole. Le titre du poème cropose ainsi la génèse de la Parole. Nous n'y lirons pourtant ni la "parole sèche", le verbe divin préexistant à la créat:on, parole indifférenciée, sans conscience de soi, ni la "parole humide" qui a germé dans l'oeuf cosmique et qui fut donné aux hommes en géné$\mathrm{ral}^{3}$. Nous suivons ici une microcosmogonie aù le verbe poétique, plutôt qu'un don, c'est une conquête alchimique, c'est la Pierre ;hilosophale que recherchent les rares adeptes de la Poésie.

Alchimie du jour nous offre done plusieurs isotopies enchevê. trées dont trois pourront être reconnues au cours de notre étude:

- la maitrise du corps, la l'bération physique suivi de l'illumination et de l'ascèse ou la réécriture de la Passion et de la resurrection du Christ;

- la transmutation des métaux;

- l'alchimie du verbe poétique.

1 Cite par hUTIN. S. Lislchimie. Paris. P.U.F.. 195I. D. 104-5.

2 HUTIN. p. 31.

3 CHEVALIER, J. G GHFFRRANT, A. Dietionnaire des smboles; mytion, rêri, costumes, xestes, formes, figures, couleurs, nombres. Paris, Seghers. 1973, v. 3 , D. 364 . 


\section{2 - LECTURE SYMBOLIQUE}

\section{1 - Préparation de la matière de l'OEuvre: la purification du sujet}

"Le plus rude travail, la peine toute entière Est à par faitement préparer la matière."

\section{Augurelli, Crysopée}

Le poème s'écrit comme un ancien livre d'Alchime où l'on voit le neophyte sous la direction d'un maitre qui l'introduit dans les mystères du Grand OEuvre. Le langage ésotérique où prédomine la fonction conative, orientée sur le destinataire, se revêt d'une forme impérative qui montre la supériorité de l'Adepte, le grand initié incarné par le je poétique, ce sujet occulte de l'énonciation qui connait déjà tous les secrets de l'art qu'il enseigne (alchimie pratique, mystique, poétique). Le destinataire du message est représenté par un néophyte designé soit par tu soit par vous. Ainsi l'axe de la communication s'oriente de manière três subtile vers un "adepto intradiégétique" (selon la terminologie gennettienne) ${ }^{4}$ auquel correspond un destinateur intradiégétique, soit vers un "adepte extradiégétique", auquel le lecteur intéressé peut s'identifier et auquel correspond une sorte "d'auteur implicite". De cette manière le poème gagne tout de suite une ambiguité féconde qui multiplie ses possibilités de lecture.

Certaines conditions préalables deviennent fondamentales pour l'accomplissement du Grand OEuvre: avant d'entreprendre le travail, tout alch:miste doit subir un processus de purification afin de pouvoir bénéficier de l'appui divin. Il doit éliminer tous les désirs corporels, mépriser et vaincre la chair. C'est justement ce que dit la première strophe du poème. Toute satisfaction physique est rechassée par la double négation autoritaire qui marque les phrases impératives; c'est le temps de l'interdiction, de la privation, de l'abstinence, du jeûne: "Qu'aucune servante ne te serve". Les macérations participent à la pénitence; en attachant la "bête de sang" qui représente le côté animal ou profane de l'homme, celui-ci se prépare pour s'élever hors de sa corruption habituelle.

Ramarquons tout d'abord l'isomorphisme qui unit les syntagmes "peine sauvage", "bête de sang", "branches basses", l'acte d'attacher aux branches infér:eures d'un arbre et la couleur noire. Nous avons affaire à un monde chtonien peuplé d'images thériomorphes que le

4 GENETTE, G. Figures III. Paris, Seuil, 1970. 286 p. 
schème de la chute relie. Le mouvement vers le bas est marqué tout de suite par le sème "avilissement" qui compose le sémène servanto au début de la strophe, laquelle finit par l'image des branches au ras du sol, suivant le même cinétisme. Les images se resserrent en chiasmes:

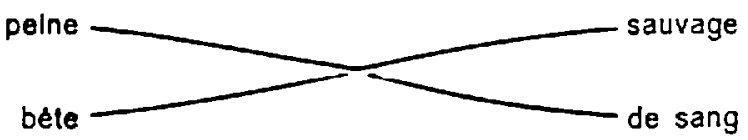

Le sang, principe corporel, apparait ici comme le véhicule des passions, comme la couleur de la libido, comme le symbole de l'instinct non controlé de l'amour mondain.

$\mathrm{S}$ : en Alchime l'arbre est le symbole de la philosophie hermétique ${ }^{5}$, le sapin du poème perd ses qualités positives parc qu'il est noir. La couleur noire rappelle en Alchimie celle du fer et du plomb, les métaux qui symbolisent la vulgarité, la lourdeur, l'inintelligence auxquelles l'homme sauvage est soumis.

Combien de temps la pénitence dure-t-elle?

Le déictique ce et l'impératif, mode de la parole actuelle, nous présente une situation très prochaine. Ces deux structures s'opposent yourtant au prétérit défini ("où tu lias") qui rejette le fait dans un passé plus lointain. Cette contrainte temporelle abolit toute chronoiogie extérieure en faveur d'une durée intérieure ou d'un espace hors du temps dans lequel le néophyte est introduit et où le temps de la pénitence ne peut être mésuré.

Quel est l'espace de la privation?

La période de privation est une période de solitude totale. Ayant laissé dehors la bête, I'homme s'enferme dans la chambre (strophes 3 et 4)), oeuf philosophique où il va subir des transformations progressives parce qu'il est lui-même la matière du Grand OEuvre. Mais le poème propose aussi la lecture de la naissance du verbe poétique. Ainsi ce Grand Adepte qu'est le poète devient luirnême l'oeuf philosophique où s'insère ef où va mûrir la materia prima verbale qui deviendra, après de lentes transformations, la pierre philosophale de ses poèmes.

On voit done qu'il s'agit d'un espace doublement emboîté: l'oeuf philophique est à la fois le siège et le sujet de toutes les transmutations.

b Cf. JUNG, G. Puicolozin y alehimia. Buenos Aires, S. Rueda, 1957. n. 40. 


\section{2 - La Putréfaction}

De la seconde à la sixième strophe nous pouvons reconnaitre, malgré les difficultés du texte, la phase de putréfaction, de dissolufion de la matière jusqu'à sa réduction à une masse informe. Cette operation, marquée par le schème de la descente, est évoquée d'une manière très subtile ef la richesse des symboles oblige à de fréquentes digressions.

Le futur hypothétique aussi bien que l'impératif négatif ne sont qu'un piège du "Grand Adepte" pour garantir l'ambiguité, l'ermétisme de son texte, où l'on doit lire soit une anticipation des étapes postérieures de l'OEuvre, soit un présent permettant d'accompagner au fur et à mesure les transformation subies par la matière.

Enfin, puisque la peine physique doit être maîtrisée par la pratique ascét:que jusqu'à ce que la douleur soit dédaignée, le présent de toutes les souffrances qu'apporte cette phase (dont on ne voit que ies conséquences, dans les strophes 7 et 8), est déguisé par son rejet dans le futur hypothétique et par l'impératif négatif. Ce présent n'est jlors suivi qu'indirectement, d'une manière invertie, à travers cette sorte de miroir construit par la fausse négation ef le transfert temnorel.

Une première lecture nous informe que personne ne doit être averti du travail du néophyte dont la solitude doit être absolue. Pouriont "les filles de feux roux" et encore d'autres apparaissent dès qu'elles sont nommées par le texte.

Le roux est une couleur qui se situe entre le rouge ef l'ocre; c'est un rouge terreux. II caractérise le feu impur, qui brû'e sous la terre. Le feu terrestre est chtonien. Les filles de feux roux peuvent êfre donc des entités démoniaques représentant la luxure, la passion du désir, la chaleur d'en bas qui consument l'être physique ef spirituel.

Le violet, fait d'une égale proportior de rouge et de bleu, est la couleur de la tempérance et symbole de l'équilibre entre la terre ef le ciel, le sens et l'esprit, la passion et l'intelligence, l'amour ef la sagesse. Le coeur, siège des sentiments dans la tradition occidentole, devient ici le centre de l'intelligence et de l'intuition, conformént les civilisations traditionnelles. C'est par là que cette image rejoint le symbolisme de la couleur violette. D'autre part, si coeur revoie d la notion de centre, il est également isomorphe de feu, foyer vital. D'où I'homologie suivante: 
SCHMIDT, z. Iecture alchimlque...

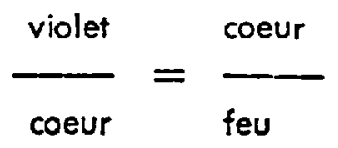

L'allusion au Tarot est très nette dans l'ensemble des strophes 2, 3 ef 4. L'arcane XIIII, nomée la Tempérance, représente un ange qui tient dans ses mains deux vases, l'un bleu, l'autre rouge, entre lesquels s'echange un fluide incolore, l'eau vitale. Le violet, invisible sur sette représentation, est le résultat de cet échange entre le rouge chtonien, et le bleu céleste ${ }^{8}$. Or, tel que l'ange du Tarot, les entités féminines du poème portent des vases, des amphores. Si la couleur violette est à peine suggérée dans le jeu de cartes, elle a ici son expression directe ef apparait justement après le rouge des filles chtoniennes ef avant le bleu dans !a troisième strophe.

La lame XIIII $\varepsilon: \uparrow$ considerée comme le syrnbo!e de l'Alchimie, la transfusion spirifulle. Cette doctrine repose sur le principe de l'échange spirituel entre le ciel et la terre par le mécanisme de l'involution - ou redescente. C'est le cycle du renouvellement périodique, mort et sublimation étant suivies de renaissance ou de reincarnation. '. Ainsi, la couleur violette et son symbolisme introduisent la mise en abyme, un procédé iconique qui ref'ète à un niveau microscophique les opérations alchimiques du poème. Quant au nombre sept, sa signification est très riche.

Le sept est le symbole de l'union des contraires, et partant il devient isomorphe du violet, qui manifeste l'union du rouge infernal et du bleu céleste, présent et dans le Tarot et dans le poème. Le sept joua d'ailleurs un rôre très important dans le Tarot: les mariages d'arcanes majeurs forment le sept. Et mariage renvoie de nouveau à l'union du rouge, cou'eur masculine, et du bleu, couleur féminine, qui entrent dans la composition du violet. Le Tarot compte vingt et un arcanes e repartissant soit en sept ternaires, soit en trois septenaires. "A l'inérieur même de chaque septenaire, les trois premiers arcanes s'opposent et le septième ramène le tout à l'unité" 8 , ce qui met en valeur la signification synthétique de la Tempérance. Le sept, signe de la croissance de la nature, rejoint encore une fois le violet, qui propose également le renouvellement cyclique. Ainsi l'arcane XIV du Tarot intimement lié au travail alchimique, est repris dans ses moindres détails par le poème.

6 Cf. CHEVALIER \& GHEERDRANT, r. 4, p. 396-7.

7 CHEVALIER * GHEERBRANT.

8 CHEVALIER \&HEERBRANT, v. 4, p. 265. 
Parce qu'il est le symbole de la résolution du dualisme, nous le rappelons, le sept est un symbole de l'unicité et par là de perfection. Ll est aussi l'expression de la Parole, mais on est encore loin de la Pórole Poétique. La matière doit subir tout un processus de putréfacfion, elle doit mourir pour renaitre après et enfin accéder à la Perfection.

Les strophes 3 et 4 sont enveloppées d'une atmosphère séraphique. Le schème ascensionnel les traverse d'un bout d l'autre: le bleu est une couleur supra-terrestre, la couleur de l'éternité tranquille ef hautaine; le verbe hisser indique mouvement vers le haut; les cheveux se situent dans l'extrémité supérieure du corps; observons cependant que les flames sont inversées, annonçant d'une manière très subtile un cinétisme descendant.

Dans le Tarot le bleu, couleur passive, est la couleur des va. leurs féminines par excellence, couleur du sentiment, de l'anima. C'est pourquoi les pitiés sont bleues. Le sème "feminite" renvoie à amphore, à coupe, contenant féminin dont le contenu est aussi féminın: l'eau et... la pitié. L'amphore par son tour est isomorphe de coeur: le coeur est souvent représenté par un triangle renversé, symbole du principe passif ou féminin. L'écriture hiéroglyphique égyptienne représente le coeur par un vase. Le coeur est aussi mis en relation avec le saint Graal, coupe qui recueillit le sang du Christ et dont le mythe a été toujours enveloppé de mystère. Le coeur est aussi un coffret où les secrets sont enfermés. Or, le bleu est également la couleur du mystère ef du secret. Les images forment donc un reseau homogène:

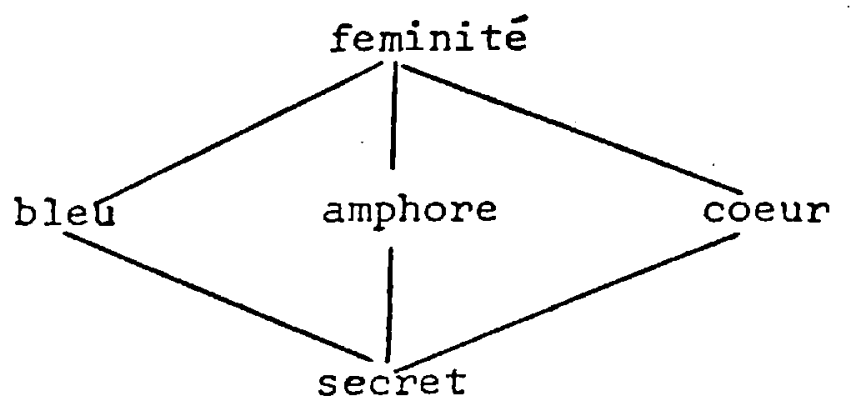


Le bleu pénètre la couleur mauve, un violet très pâle ef lui em. crunte son atmosphère d'irrealité voire de surrealité. Et "entrer dans le bleu, c'est un peu comme Alice au Pays des Merveilles, passer de l'autre côté du miroir" ". Cela explique l'image inversée de flammes marines ("ombres mauves pareilles à l'envers des flammes narines".) Par son irréalité le bleu résout en lui-même les contradictions. Ainsi devient logique la combinaison antithétique du feu et de l'eau dans l'image des flammes marines. Cependant le processus d'inversion amène la perte du caractère sexual du mariage de ces deux éléments: des flammes inverties sont des flammes châtrées. En outre la transparence de la couleur bleve qui teinte les ombres mau. ves déréalise le feu. Sa fraicheur aérienne refroidit les flammes. Elle !eur ôte aussi un mouvement plus intense, Sans l'élément mâle, la mer, symbole du coeur humain en tant que siège des passions, devient elle zussi stérile. "L'humidité chaude" n'y est plus possible.

Le sème "apaisement" réunit plusieurs sémèmes éparpillés dans

ies strophes 2, 3 et 4: calme, tranquille, coeur violet (= amphores aux pifiés bleues), bleu, mauve. Soulignons en passant que la mauve est une plante herbacée à fleurs d'un violet pâle dont l'infusion est calmante.

Les adjectifs calme, tranquille, longue, processionelle, le substantif frise et le verbe plisser se partagent le sème "lenteur", isonorphe de l'apasement.

L'isotopie religieuse marque le bleu, signe de la Vierge, couleur ascétique dont la profondeur a la même gravité solennelle d'une "cal. me procession". Ainsi le bleu, le mauve ef la "calme frise processionelle" appartiennent au nême champ sémantique, sans songer au violet, couleur de la Passion du Christ.

Dans ce climat surnaturel, tout près de l'infini, la chambre du léophyte devient soudain une chambre cosmique avec "les quatre vents de ses murs". Le quatre est un nombre cosmologique, totalisa. ieur, archétypique, sacré. Quatre, nombre des éléments, est le nombre des portes que doit franchir l'adepte de la voie mystique selon une tradition orientale. Pour les alchimistes, la quaternité est un axiome fondamental dans la recherche de la pierre philosophale.

L'on rejoint ainsi le contexte alchim que du poème qui permet de compléter la lecture des strophes 2,3 et 4 . 
Le schème ascensionnel, les couleurs aériennes et évanovissantes qui confèrent à ces strophes un climat vaporeux nous font penser à la phase de volatilisation, la troisième reconnue par la "voie sèche" appliquée par les alchimistes de l'Europe occidentale, qui voyaient dans l'OEuvre sept opérations. ${ }^{10}$ D'autre part l'évocation indirecte des filles de feux roux nous suggè,e que le feu de l'Athanor a été allumé, marquant le début d'une série d'opérations inaugurées par ce "Régime de Mercure", le premier des sept "régimes" de Philalethe: à plusieurs couleurs nuancées (strophes 2,3 et 4 ) se succède le vert (strophe 5); puis apparait la couleur noire (suggérée par le cromatisme sombre de la sixième trophe), introduisant un nouveau régime, le "Régime de Saturne". 11

Le vert tonifie la cinquème strophe ("filles aux pieds de feutre vert", "pré", "herbes"). La liaison entre celle-ci et les strophes précédentes se fait à travers le passage du violet au bleu puis au vert, suivant les couleurs de l'arc-en-ciel, couleurs intermédiaires par lesquelles passent les principales opérations alchimiques, caracterisées par le noir, le blanc et le rouge. Un même symbolisme relie le violet au vert: toutes les deux couleurs représentent le renouvellement cyclique. Le vert, comme le violet, c'est une couleur médiatrice. Equidistant du bleu céleste et du rouge infernal, il résout les antagonismes entre le haute et le bas, le froid et le chaud. Au bleu cáleste se succède le vert terrestre, une couleur plus humaine, plus tiède, plus enveloppante, reposante et nourricière. D'où l'isomorphisme des adjectifs vert, doux, dru, lent, silencieux et le substantif feutre. Par conséquent l'espace vert aux "herbes silencieuses" exclue "I'espace vif du cri": dans 'Jn espace rassurant e thérapeutique, où le soleil est moins pénible parce qu'il est "tondu", c'est-à-dire, parce qu'il a ses rayons coupés, les pe nes sont soulagées, les cris des douleurs physiques et morales cont étouffés. Mais, remarquons d'ores et déjà que le cri a été nommé. Signalons en passant que la tonsure du soleil nous offre l'image du "soleil dans le soleil" qui rappelle le signe alchimique de l'or (dessiné f!us loin).

Le vert, la couleur de la régénération, est associé au mythe du Christ. Les peintres du Moyen Age peignaient en vert la croix, instrument de régénération du genre humain, assurée par le sacrifice de Jésus ${ }^{12}$ Or, nous retrouvons dans cette stro. phe l'isotopie religieuse. Les antiques tapis "réservés au rérou-

10 HUTIN, p. 122.

11 HUTIN, p. 121.

1: CHEVALIER \& GHEERBRANT. v. 4, n. 376. 
lement lent des douleurs sacrées" nous font penser à la via crucis. Rappelons que la couleur violette avait déjà annoncé la Passion. Le vert, par son caractère médiateur, évoque un rite de passage qui prend des dimensions cosmiques: le soleil devient anthropomorphe ci, comme les prêtres et les moines, passe par le rite de la tonsure, signe de renonciation à la chair. On peut alors ajouter que le vert est la couleur de la connaissance spirituelle.

Enfin, l'isotopie de l'écriture nous montre que, si l'on a su dépasser l'âge du cri, cette parole primitive, on l'aperçoit quand même au loin, puisqu'il a été nommé.

A partir du passage du bleu au vert, les couleurs s'assombrissent. Au bleu céleste se succède le vert terrestre et à partir de là le cinétisme de la descente et le processus de décompasition seront intensifiés.

La strophe 6 nous suggère la descente dans les abimes de l'être. Cet espace chtonien, "obscure" ef "souterrain", est le siège des passions sauvages. Cet aspect est mis en relief par le vocabulaire hyperbolique (obscure, souterrain, forte vibration, passion excessive) ef par l'image de l'amour cosmique dans la comparaison entre la passion volcanique et la mer des passions.

Le monde des profondeu's telluriques désigne le chaos ténébreux des origines, les états informels de l'existence. II retrouve par là le symbolisme de la mer, de l'océan qui, en raison de leur étendus apparemment sans limites, sont les images de l'indistinction primordiale, de l'indéterm nation principie!le. Cet aspect enrichit la signifi. cation du lexème appareillant: l'isotopie ma-ine de la strophe permet de comprendre le verbe appareiller, dont est issu l'adjetif, comme synonyme de "lever lancre", "se disposer au départ", ce qui le rapproche du champ sémantique du nom origine, "d'où sort toute chose"; or, si l'origine est le lieu de l'indistinction primo-diale, appareillant peut être "tout pareil".

Ces observations nous reconduisent à l'isotopie alchimique. II faut que la matière de l'OEuvre soit ramenée "à un état de simplicité indifférenciée, comparable à celui de la matéria prima... afin qu'il soit apte à recevoir la vib.ation du fiat lux initiatique" (René Guerion) ${ }^{13}$. Cette décomposition de la matière, cette descente dans les frofondeurs de l'être, correspond à la phase de putréfaction qui esł pourtant bien nécessaire à la progression ultérieure. La graine doit être ensevelie dans le sein de la terre, doit pourrir pour renaitre.

Cite par HUTIN, p. 98. 
Ainsi l'Adepte doit traverser "la nuit noire de l'ame" pour mesurer son indignité et obtenir ensuite "la sublime transmutation du charnier natal, immonde matière noire et faire du charbon un éclatant diamant, du plomb vil un or pur." (Paracelse) ${ }^{14}$

Quant à l'isotopie de l'écriture, elle nous dévoile le profil indistinct de la Parole primordiale, ontologique, avant le cri (strophe 5), "en l'origine de son chant apparefllant", qui est plutôt un bruit.

La phase de putréfaction est donc à sa limite, et tenant compte des isotopies analysées, nous pouvons la représenter par le tableau suivant:

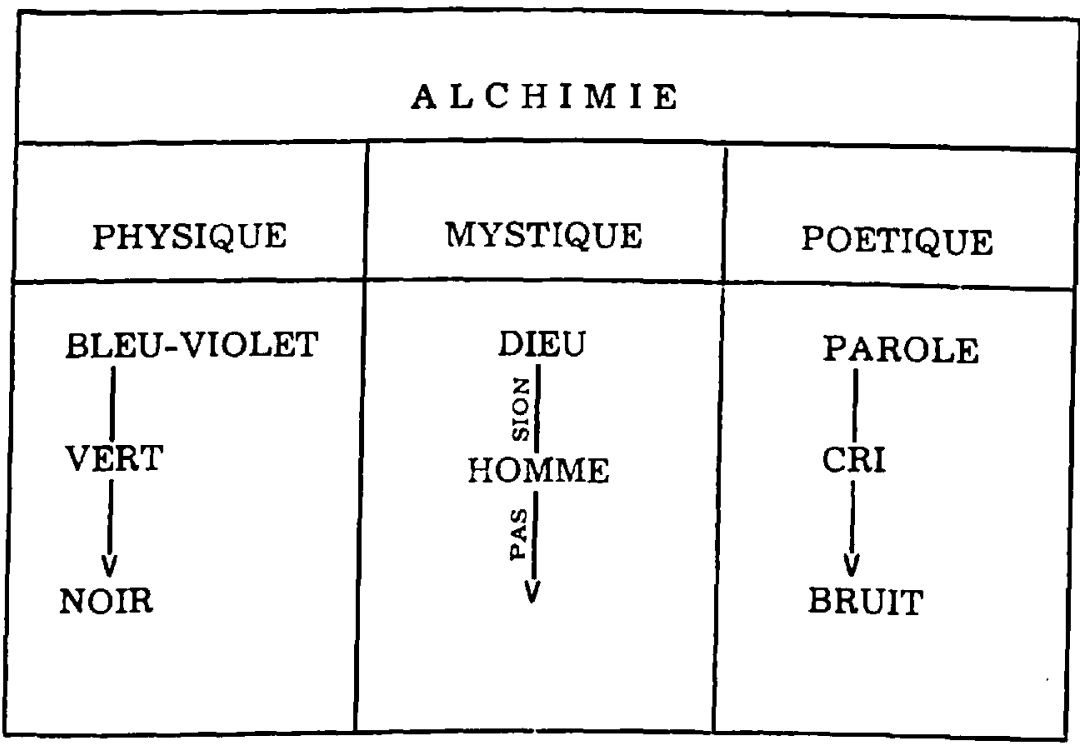

\section{3 - La Rósurrection of l'Ascèso}

La phase de mortification, de putréfaction, "la plus sombre" et "la plus lente" de toutes les opérations alchimiques est rappelée dans les strophes 8 et 9 a travers l'allegorie de deux filles: "la plus sombre des soeurs" ef "la plus lente d'entre elles". Mais la mortification est nécessaire à la floraison de la sagesse; la pourriture est indispensable à une germination normale. Ainsi, une thérapeutique symbolique (baumes, remèdes, conseils noctumes, strophe 8 ) et des :mages végétales (strophes 7 et 8 ) annoncent la réssurection posterieure de la matière.

14 HUTIN, p. 99. 
La strophe 7 reprend et développe la méraphore de la première. Le mot soeurs, avec sa connotation religieuse, est lié au mot servante qui peut être lu maintenant dans la même isotopie ("servante de Christ, femme qu: sert Dieu fidèlement'); amarrer est synonyme de lier; l'amour blessé explique peine sauvage et bête de sang; le sang est isomorphe des veines ouvertes et, par une sorte de contagion métonymique, les branches du noir sapin se teintent de rouge et s'épanouissent en feuillages de vaisseaux sanguins.

La mortification du sujet est maintenant accomplie. "La chute est finie. La mort commence." 15

Cette phrase de Bachelard nous montre bien qu'en Alchimie la mort n'est jamais totale, absolue. Le sémantisme du ve:be commencer, au présent, ne pose pas un processus accompli, mais plutôt un renouvellement cyclique. La mort n'est que la dissociation des êtres et "leur retour dans le corps leur mère" (Paracelse). ${ }^{16}$ C'est pourquoi, dans le poème, le sujet n'est jamais perçu comme ent èrement mort, malgré ses blessures graves. L'image des "fevillages de tes veines ouvertes", née du rapprochement du sang, symbole de la vie et du sapin noir, isomorphe de la mort, du cercueil qui pourtant rappelle le berceau, est une image dynamique où coule un sang encore chaud et qui prépa:e l'image des "baumes nouvellement fleuris" de la strophe suivante.

Cette image est doublement revifiante: le baume est un liniment, qui "rend de nouveau la vie"; en outre la vie bourgeonne dans les baumes fleuris du poème. Et si l'on reprend successivement le noir sapin de la première strophe, les feuillages (strophe 7) ef les baumes nouvellement fleuris (strophe 8 ), on verra un noir sapin baumier qui renâit. Cette image végétale fleurissante préfigure la résurrection de la matière de l'OEuvre.

Les baumes, aussi bien que les soeurs, renvoient à l'isotopie mystique. Dans les cérémonies religieuses, le baume signifie le parfum de la grâce ou la bonne odeur que doivent répandre les vertus du chrétien; l'Agnus Dei est béni avec du Saint Baume. L'allégorie des soeurs accueillant le Mortifié avec des baumes rappelle les femmes pieuses de la Galilée qui ont voulu oindre le corps du Crucifié. " Alors, l'arbre ranimé du poème rejoint tout naturellement la croix, symbole ascensionnel et cyclique.

16 Bachelakd, G. La terre et lea réreriew de la volonté. Paris. Corti, 1948. p. 402. 16 Cité par HUTIN, p. 61.

17 Cl. Marc 16-1-2; Lue $21: 1$. 
Le lexique des strophes 8 et 9 montrent un cinétisme descendant suivi d'un mouvement ascendant. L'isomorphie des schèmes dans les deux strophes attestent la parenté des soeurs:
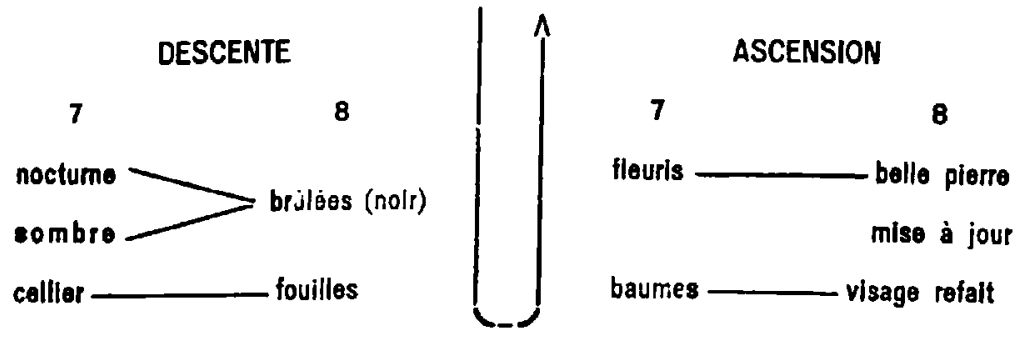

II est intéressant de remarquer, dans la strophe 9, une sorte de "technique du miroir", annoncée dans la quatrième stroptre: lo transformation du-sujet, de la matière n'est observée qu'indirectement, à travers le visage de la soeur la plus lente (elle "referait son visage.... $)^{\prime \prime}$.

\subsection{1 - Le Petit Magistère}

La matiè e de l'OEuvre est complètement dissoute. II n'en reste que les sels, principe substantiel des choses, essence première, sécrète, "graine du monde", esprit vital, substance incorruptible qui sera la matière prochaine de la Pierre Philosophale.

Les strophes 10 et 11 déploient cette rêverie substantialiste, annoncée dans la neuvième strophe par les "fouilles patientes et pu res" et surtout par l'image des "larmes brûlées".

La récolte de la substance première réclame "des paniers fins" et ne peut être fa'te que par "une fille de sel" en miniature dans "le jardin qui s'affale" celui-ci est le developpement métaphorique de la matière pourrie, fondement et engrais de toute moisson.

La substance concentrée se manifeste sémiologiquement par le sel, les pleurs (qui reprend la métaphore des "larmes brûlées"), la rosée jusqu'aux détails des paniers fins el des moissons claires, finesse et transparence étant des qualités d'une matière purifiée.

En tant qu'essence de la douleur humaine, "suc" de l'homme conienant elles mêmes du sel, essence piemière, les larmes sont une substance doublement concentrée. Les plours prennent ensuite des dimensiones cosmiques et deviennent rosée. 
La rosée est liée aux mythes de la fécondité. Elle correspond à "la panspermie de l'atmosphère" 1s. Fabre, cité par Bachelard, considere la rosée comme

Une gelée très délicate de la quintessence de tous les éléments, et du plus pur des influences célestes qu'elle mêle ensemble, et en fait une liqueur propre à nourrir toutes choses. La rosée est une liqueur élémentaire qui enferme en soi les vertus et proprietés de toute la nature. ${ }^{19}$

Parce qu'elle résout l'opposition des eaux d'en haut et d'en bas, ies eaux terrestres et célestes, elle s'approche du sel, principe méd ateur de deux substances complémentaires.

La vertu purificatrice du sel rejoint celle de la rosée, expression de la bénédiction céleste et de la grâce vivifiante. Pour les alchimistes, cette quintessence astrale recueillie ou retrouvée dans la P.erre philosophale permettait d'obtenir le germe de la pureté ef faisait da la Pierre la condensation de tovies les grandes substances du ciel, de la terre et de l'eau.

La rosée, les pleurs et le sel ainsi reliés forment dans les poème une pussante combinaison régénératrice de la matière pourrje.

La rosée est aussi le symbole de la parole divine. Le cantique Je Moise (Deutéronome, 32) débute par son évocation: "Que ma doctrine ruisselle comme la pluie, que ma parole tombe comme la rosée..." "2n. La rosée "est vraiment de l'aube distillée et fruit même du jour naissant." 21 .Cette qualité de l'eau céleste et son somorphis. me avec la Parole renvoient au titre "Alchimie du jour" et fait de la rosée la synthèse de toutes les isotopies alchimiques du poème.

La rêverie substantialiste se prolonge dans la strophe suivante par la voie de l'isolopie religieuse.

Véronique est la femme pieuse qui aurait essuyé le visage de Jésus, couvert de sang et de sueur, lors de la montée au Calvaire. Le linge garda l'empreinte de la face divine. Vouloir saisir "la grimace" d'un visage empreint, c'est vouloir fixer le spectre d'un être, son reflet. son essence. Voir dans les voiles déroulées des clairs miroirs d'un équivaut à retenir la transparence. à capter la fluidité.

Si la strophe précédente offrait l'image des pleurs, une substance 
doublement concentrée, comme nous avons vu, dans cette strophe rous trouvons des images doublement réfléchies: il s'agit des miroirs (le visage, miroir de la grimace; les voiles, miroirs de l'eau) dans les niroirs (les toiles, miroir du visage; l'eau, miroir des voiles).

Obtenus les sels très purs de la matière dissoute à travers l'opératión appellée distillation, évoquée dans le poème par les images des larmes et surtout de la rosée, le feu de l'Athanor doit être maintenant gradué, parce que les sels cristallisés doivent être décomposés par la chaleur. Ainsi "se hâte la fille-fièvre parée d'epines cuivrées" (strophe 12). La fièvre et la couleur cuivrée, une couleur rougeâtre, rejoignent l'isomorphisme du feu. Pour Paracelse, le feu, c'est la vie et qui recèle du feu a vraiment le germe de la vie. ${ }^{22}$ Les épines cuivrées dont l'entité féminine est parée rappelle encore une fois la figure du Christ, le Ressuscité. Le feu et le Chrisł dans colte allégoré a!chimique annonce la Résurrection proprement dite de la matière de l'OEuvre, avec l'obtention de la couleur blanche, sous le "Régime de la Lune" (Philalethe). Enfermée dans l'Oeuf, la matière meurt, noir $c^{i} i$, puis renait en perdant sa noirceur. "En des miracles certains de couleur ag'le" (strophe 15), c'est-à-dire, en prenant plusieurs tonalités, la matière devient progressivement blanche ef de nouveau dissoute par "I'eau puissante" (strophe 15), par "l'eau forte", c'est-à-dire, par lacide nii:ique, elle se convertit en bove d'argent. Avec la coagulation. apparait la pierre blanche, "la blanche floraison d'une perle dure" (strophe 13).

Symbole lunaire, la perle est associée à l'eau et à la femme. Par conséquent "la perle dure" n'est autre que la pierre blanche des a'chimistes, capable de changer les métaux imparfaits en argent, métal symbolisé par la Lune et riche en principe mercure, principe fémin'n upparenté à l'eau. La perle se relie donc aux larmes ef à la rosée des strophes précédentes. Selon les légendes, d'ailleurs, la perle nait par la chute d'une goutte de rosée dans la coquille, qui devient un véritable oeuf philosoph que où la substance sera cuite, digérée et faite perie.

Le symbolisme de la Parole est isomorphe de celui de la perle: la coquille est le langage et la perle qu'il contient est le sens secret du langage ou - ajoutons - la parole poétique.

De même que la Vie est fille de la Mort, le Jour est fils de la Nuit. La nuit symbolise ici (strophes 12 et 13) l'espace des ténèbres où fermente le devenir, le temps de la maturation ("paumes mures de 
la nuit"), des germinations qui vont éclater au grand jour, le temps de la gestation de la Pierre, gestation qui peut durer vingt-quatre heures (ie poème semble accomplir ce cycle) ou des siècles: "Sur tes paupières bientôt elle posera ses mains étroitement comme des huítres vives où la mort médite, des siècles de songe sans faille, la blanche fioraison d'une perle dure" (strophe 13).

Le temps de la couvaison, de la méditation doit être "sans faille": le feu de l'Athanor ne doit pas cesser de bruler jusqu'à la fin de I'OEuvre; l'alchimie transcendante impose à ses adeptes une ascèse bien reglée.

L'image de l'héliotrope dans la comparaison anthopomorphe de la nuit ("la nuit, en sa haute taille levée, bouge des paumes mûres comme des noirs tournesols'), évoque la conjonction des ténèbres et de l'élement solaire. Autrement dit, la nu't garde le germe de la journée, elle est le commencenent du jour où jaillira la lumière de la vie-journée, pierre, sagesse ou parole. Cette idée est renforcée par la conjonction des sèmes de la vie et de la mort dans un espace où cesse toute opposition ("huitres vives où la mort médite").

Le reseau d'images est donc très bien tissu. Les équivalences sémantiques sont très riches, ce qui contribue à l'unité du poème. Voyons encore quelques exemples dans les strophes 12 et 13:

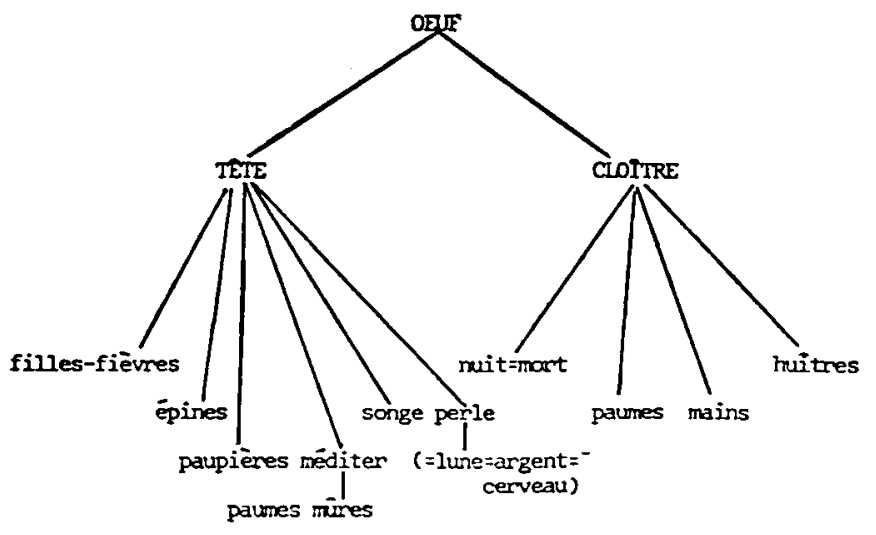


La matière a ressurgi. Donc elle aura son ascèse (strophe 14): hissée comme un drapeau ("O toi qui trembles dans le vent") à un mât cosmique qui rejoint le symbolisme du renouvellement cyclique ("au mât des quatre saisons"), la pierre blanche expose son beau visage. "Nue", c'est-à-dire, pure, (la perle est blanche et reputée sans défaut), elle est consacrée par l'onction, comme Jésus (Christ, du grec $k$ ristos, signifie "l'Oint", "le Roi"). Elle "grince de sab'e", rappelant que sa matrice, l'Oeuf philosophique, a été mise au four sur une écuelle pleine de sable. Le sable peut aussi se substituer à l'eau, élénent purificateur, liquide comme l'eau, abrasif comme le feu. Dans ce cas il se relie aux huiles pures dans le rite de consécration. Mais comme tout symbole est polivalent, les grains de sable peuvent signifier les péchés dont la mat ère se défait. Rappelons la connotation négative du sémantisme de grincer, "produire un son aigu, prolongé et désagréable".

Si le traveil alchimique s'arrêtaił là, on aurait tout simplement le Petit OEuvre ou Petit Mag stère, qui se bornait à l'obtention de la pierre blanche permettant de faire de l'argent. Mais, "redoute l'avènement silencieux des compassions crayeuses aux taces d'argiles brouil. lés", avertit le Grand Adepte. Méfie-toi de la fragilité de la cra:e (minaral d'origine animale) et de l'argile (d'origine végétale), les premiers en ordre de destruction d'entre tous les autres minéraux fossiles du vant. ${ }^{23}$ Autreme, it dit, il taut crainare ta puritication superficielle, le faux perfectionnement. L'OEuvre véritable continue le chemin vers l'essence des choses. Il ne faut pas se limiter à faire de l'argent si l'on jeut obtenir de l'or.

\subsection{2 - Le Grand Magistère}

La matière doit poursuivre son lent travail de maturation (strophes 17 et 18). Par la coction au grand feu, "le vif pouvoir" (strophe 17), "en des équipages fougueux" istrophe 18), commence l'opération appelée rubification. La pierre devient progressivement verte, blue et rouge foncé ("Pose le vert contre le bleu... ne crains pas l'ocre..."), correspondant au "Régime de Vénus" de Philalethe. Enfin, sous le "Régıme du Soleil". "apparait le rouge éclatant, le rouge parfa't: "ne crains pas l'ocre sur le pourpre." Et puisque "les belles couleurs scint des signes de pleine maturité", ${ }^{24}$ on a trouve la Pierre philosophale, la jierre au rouge.

L’Oeuf est brisé: "Les murs aux tessons bleus crèvent comme des

2. BACHELARD, D. 267.

24 BACHELARD, p. 247. 
cercles d'eau sur la mer" (strophe 20). Le substantif tessons, la couleur bleve et l'élément liquide (eav, mer) suggèrent parfa'tement l'image du petit ballon en cristal qui se brise. La Pierre rouge (coeur) a pris la forme de sa matrice: "E† le point du coeur dessine sa propre souple ceinture" (strophe 21). Et aussi le forme de la Perfection.

Les noyaux circulaires des images (point coeur, cercle, ceinture) dessinent le symbo!e alchimique du Soleil, de l'Or:

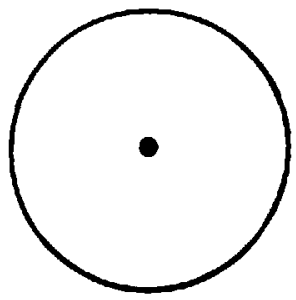

Le cercle, deuxième symbole fondamental ${ }^{2 \pi}$, se manifeste dans ie poème par le lexème ceinture et, de manière explicite, dans l'mage "des cercles d'te"" zur la mer". Ces cercles concentrique: "tprésentant les degrés de l'être, les hiérarchies créées, jouent le rôle d'une conclusion qui rappele joutes les phases de l'OEuvre, jusqu'à l'étape ultime du perfectionnement intér.eur. Sans commencement ni fin, le mouvement circulaire est parfait.

Le cercle participe de la perfection du point. Tous les deux ont des propriétés symboliques communes. Le point est le Centre, l'or:gine, le foyer. le principe et le terme de toute chose. Le point désigne la puissance créatrice, les perfections cachées, la résolution des tendances antagonistes, l'équilibre, l'harmone, l'unité primordiale, bref, Dieu. Pascal, citant Hermès Trismegiste, definit Dieu comme "une sphère dont le centre est partout et la circonférence nulle part." 26

Cercle, centre, point, voilà des symboles de la Perfection. Voilà la Pierre philosophale. Le Grand Oeuve est accompli. Poétique.

Il faut maintenant revenir au Grand Magistère de la Parole

25 Lea quatre symboles fondamentaux lle carté. la croix. le cercle et le centrel cont representes dans le noème: le carré - srmbole de la terre. de l'homne. Hes nin. tre sléments eat représenté par l'imace de la chambre du néunhyto "“los nuatro vents de tes murs") et par le nombre f. cité deux fois: la croix mt sugaérée par limare du Chriat et te larbre.

26 CHEVALIER \& GHFERBRANT. ․, 1. p. 299. 
Le verbe qui a été donné aux hommes (verbe = don, strophes 17 et 18), devient la matière d'une alchimie verbale.

Par la destructuration du langage, le verbe descend jusqu'aux abimes du cri et du bruit. Ayant été suffisamment cuit dans l'Oeuf, le ve:be ressurgit du silence et peut maintenant briser son cloître et se lier au monde, comme l'a fait Jésus-Christ qui s'est introduit parmi ses disciples après la Résurrection. ${ }^{2 T}$

Dans la strophe 17, verbe, fldche ef lumière s'associent en une seule image ("laisse débonder le verbe se liant au monde telle la fiècle à son arc"). La flèche est le symbole de la pénétration, de l'ouverture. L'orifice est une lumière. Ainsi le verbe est la flèche qui ouvre le monde et la lumière qui le pénetre pour le féconder. Telle une flèche ou un éclair, le verbe poétique liberé "profère des choses sauvages dans le soleil" ef "nomme toute chose" recréant le monde dans une nouvelle cosmogonie, "face au fumulte des grands morts friables et irrités" (strophe 19). Ne pouvait-on pas y voir un poème nouveau bouleversant la tradition? Mêlé au soleil, le verbe poétique peint l'enthousiasme de sa naissance dans un tableau doublement lumineux parce qu'il est lui-même clarté.

Si le soleil est l'expression visible du verbe divin, cette nouvelle lumière proposée par le poème est l'expression "lisible" du verbe poétique. Comparé à l'acte créateur, l'acte de nommer élève le poète au niveau de Dieu. En fait, si Dieu a convoqué le jour par son Fiat Lux, le poème, dans une nouvelle génèse, convoque le jour "pour la seconde fois" pour engendrer de nouveaux mondes avec son nouveau verbe.

Il faut soul'gner que le cycle de 24 heures suivit dans le poime ice jour, au premier vers la nuit, strophe 12 , le soleil et le jour, strophes 19 et 22) devient une source de différentes lectures. Ce cycle peut être le temps de la gestation d'un nouveau jour. Ainsi le poème "Alchimie du jour) sera lu naivement dans un premier niveau référentiel. Il se peut qu'un poème se fasse également dans cette péirode de temps. Mais comme le temps de toute création ou de tout perfectionnement spirituel ne peut pas être mésuré parce que c'est une durée intérieure, le cycle de 24 heures peut englober plusiours cosmogonies.

Revenant à l'isotopie de l'alchimie physique, la Pierre philo. sophale, rouge et parfaite, doit être mêlée à l'or fondu pour aug. 
menter indéfiniment en qualité et en quantifé. Dans la strophe 19 on voit dèjà cette combinaison.

llluminé ef illuminant, le verbe "monte comme un large pavot

éclatant sur sa tige" (dernière strophe). C'est l'ascèse, le rayonnement ef la floraison de la parole.

Résultat d'une alchimie intérieure, la floraison, en même temps qu'elle est une manifestation extérieure signifiant l'épanouissement de la parole, elle est aussi le retour au centre, à l'unité, à l'état primordial, et l'on retombe dans l'isotopie mystique.

Si la Parole est la lumière qui engendre des réalités nouvelles, elle est aussi pavot, la Pierre philosophale rouge et l'opium qui entraine des rêveries infinies.

\section{3 - CONCLUSION}

Prenant le Verbe, matière première du poème, un Grand Adepte sépare et purifie le registre quotidien et le régistre d'une poétique tradtionnelle. Enfermé ensuite dans un espace doublement emboité, le langage est désarticulé et descend jusqu'aux abimes du cri et du silence.

Solve et coagula. Le feu est allumé. Le Grand-Oeuvre commence. A l'intérieur de l'oeuf la matière se transforme progressivement. C'est une lente cuisson. La rédaction s'insinue. D’autres rédactions se succèdent. "Pose infiniment le noir contre le blanc", dirions-nous. Et soudain la Parole brise l'oeuf. Enfin l'essence poétique teinte la page. Le Verbe ressurgit sous une nouvelle structure. L'Adepte a rouvé la pierre philosophale. Le Poème est là. Il s'appelle Alchimie du Jour.

Parce qu'il est véritablement un oeuvre d'art, il permet un nombre infini de lectures. Nous y avons perçu quelques-unes: la naissance du jour, l'alchimie physique, l'alchimie mystique, l'alchim e verbale. Et puisque le poème est lui-même une sublimation alchimique du Grand Magistère hébertien, il propose encore un autre texte: la lecture de sa propre génèse. 


\section{RESUMO}

A partir da análise semântica do poema Alchimie du Jour, de Anne Hébert, pode-se chegar a uma leitura simbólica onde se desencadeiam todas as etapas de um verdadeiro processo alquímico. Além da leitura referencial do nascimento do dia, três outras isotofias podem ser percebidas no decorrer da análise: a alquimia física, a alquimia mística e a alquimia poética.

\section{BIBLIOGRAPHIE}

1 BARTHES, Roland. Analyse textuelle d'un conte d'Edgar Poe. In: Somiotique Narrative et textuelle. Paris, Larousse, 1973. p. 29-54.

2 BACHELARD, Gaston. La Poétique de l'espace. Paris, Presses Universitaires de France. 1957. $215 \mathrm{p}$.

3

$409 \mathrm{p}$. La Terre et les rêveries de la volonté. Paris, Cortt, 1948.

4 L'eau et les rêves. Paris. Corti, 1942. 265 p.

5 . La psychologie du feu. Parls, Gallimard. 1949. 184 p.

6 Bíblia SAGRADA. Rio de Janeiro, Sociedade Biblica do Brasil, 1968. $309 \mathrm{p}$.

7 CHEVALIER, Jean et GHEERBRANT, Alain. Dictionnaire des symboles; mythes, rêves, coutumes. gestes, formes, figures. couleurs, nombres. Paris, Seghers, 1973. 4 v.

8 DictionnalRe Larousse du XXe siècle. Paris, Larousse, 1929. 6 v.

9 DURAND, Gilbert. Les structures anthropologiques de l'imaginaire. $\mathrm{Pa}$. ris Bordas, $1969.550 \mathrm{p}$.

10 FRYE. Northorop. Anatomia da critica. S. Paulo, Cultrix, 1973. 362 p.

11 GENETTE. Gérard. Figures Ill. Paris Seuil. 1970. 285 p.

12 Hebert, Anne. Poèmes. Paris, Seuil. 1960. 110 p.

13 HUTIN, Serge. L'Alchimle. Paris P.U.F. 1951. 128 p.

14 JUNG G. Psicología y alchimia. Buenos Aires, S. Rueda, 1957. 501 p. $501 \mathrm{p}$.

15 PAGE Plerre. Anne Hébert. Otawa. Fides. 1965. $189 \mathrm{p}$. 